\title{
Editorial
}

\section{¿UNSAFE ABORTION EN CHILE?}

Del 23 al 24 de junio de 2008, en Lima, Perú, FIGO convocó a las Sociedades de Obstetricia y Ginecología sudamericanas para que presentaran el diagnóstico de situación del aborto en sus respectivos países, dando marcha a la iniciativa mundial de FIGO para la prevención del aborto inseguro, enorme y dramático problema de salud pública, especialmente en países no desarrollados. Los países sudamericanos presentaron su realidad, mostrando en general, altas tasas de mortalidad materna por aborto séptico, como consecuencia del embarazo no deseado y propusieron un plan de acción, para que junto a su respectivo Ministerio de Salud, pudieran reducir los indicadores de morbimortalidad materna por aborto inseguro, de acuerdo a la legalidad vigente de sus respectivos países (1).

Las experiencias de los países sudamericanos contrastaron con la de Chile, que mostró un significativo descenso de la muerte materna por aborto desde que se introdujeron en el país los métodos anticonceptivos. En la Figura 1, se observa la evolución de la transición demográfica chilena desde 1990 al 2004. En 1900, Chile tenía un crecimiento natural de su población muy bajo, producto de una alta tasa de mortalidad, pese a la elevada tasa de natalidad. En la medida que baja la mortalidad, manteniendo una alta natalidad, el crecimiento natural de la población aumenta y alcanzó en la década de los 60 su máxima expresión. La introducción de los métodos anticonceptivos, en esa década, permitió reducir la natalidad, que junto al continuo descenso de la mortalidad, hicieron que en la actualidad Chile se encuentre en la etapa de transición demográfica avanzada, caracterizada por un bajo crecimiento natural de la población, producto de una tasa de mortalidad baja y estable, y de una tasa de natalidad moderada a baja y en descenso (2). Paralelamente, la mortalidad materna inició una significativa y continua reducción, especialmente por la disminución del aborto séptico, consecuencia del embarazo no deseado (3).

En 1960, en Chile, hubo 845 muertes maternas (tasa de mortalidad materna: 299/100.000 nv), de las cuales 302 fueron relacionadas al aborto (tasa de mortalidad materna por aborto: 107/100.000 nv). El progreso del país en sus diferentes ámbitos hizo que en 2005, la mortalidad materna fuese de $19,8 / 100.000 \mathrm{nv}$ (48 casos) y la tasa de mortalidad materna por aborto de $0,8 / 100.000 \mathrm{nv}$ ( 2 casos) (4), esta última, estable y bajo 1,5/100.000 nv desde $2001(5,6)$. Es importante destacar que la muerte materna por aborto fue una de las tres causas que presentaron una significativa reducción entre 19902000, que permitió a Chile cumplir con el Plan Regional de Reducción de la Mortalidad Materna (7). De acuerdo a las estadísticas presentadas, es posible concluir que actualmente en Chile, la mortalidad materna por aborto séptico, no es un problema de salud pública.

Pese a lo anterior, surge la legítima inquietud si las estadísticas nacionales son confiables. Es posible que en Chile, exista un subregistro de muertes maternas, incluidas las por aborto, situación que también alcanza a los países desarrollados. En el Reino Unido, en el período 2000-2002, hubo un subregistro de $44 \%$ más de muertes maternas que las registradas oficialmente por el sistema de registro civil británico (8). Un estudio efectuado simultáneamente en 2 países europeos (Finlandia y Francia) y 2 estados norteamericanos (Massachusetts y Carolina del Norte), en el período 1999-2000, mostraron un subregistro de muerte materna entre el 22 y 93\% (9). Otro estudio efectuado en Maryland, EEUU, entre 1993-2000, encontró un subregistro de $38 \%$, destacando el $100 \%$ ( 2 casos) de subregistro de muertes maternas por aborto terapéutico (10).

En países en desarrollo de la Región, el subregistro de muertes maternas también es importante. En Argentina, en 1990, en una localidad de Buenos Aires, se detectó un subregistro de $50 \%$ de muertes maternas, muchas de ellas relacionadas al aborto séptico (11). En Pernambuco, Brasil, en el período 1994-2003, la subnotificación fue de $46 \%$, siendo las muertes maternas relacionadas al aborto ilegal 


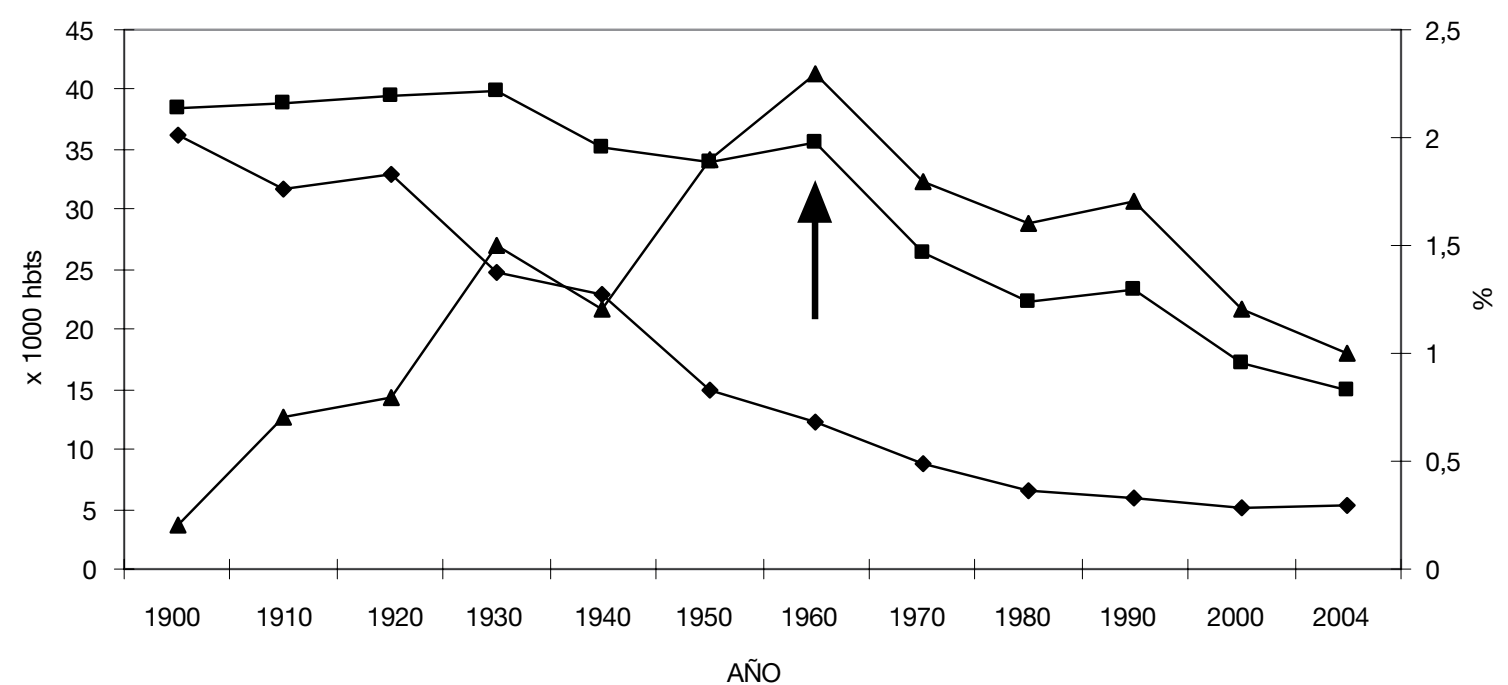

$\longrightarrow$ natalidad $\multimap$ mortalidad $\multimap$ crecimiento natural

Figura 1. Transición demográfica, Chile 1900-2004 (flecha indica momento de incorporación de los métodos anticonceptivos).

un importante componente (12). En Jamaica, en 2000 , el subregistro fue de $20 \%$ y las muertes maternas ocurridas en el primer trimestre del embarazo fueron un factor relacionado significativo (13).

Chile pertenece al grupo $A$ de registro de muertes maternas, lo que significa que tiene un sistema de registro civil de los fallecimientos, con al menos un $90 \%$ de muertes maternas registradas y menos del $20 \%$ sin exacta identificación de la causa de muerte (14). Sin embargo, la literatura muestra que los países con sistema de registro civil de las muertes maternas, pueden aumentar hasta casi en un $200 \%$ con un sistema de vigilancia activa (15). Si aplicamos a Chile ese porcentaje, para obtener el rango superior de incertidumbre en la estimación de la tasa de mortalidad materna, tenemos que para el año 2005 el rango de la tasa de mortalidad materna para aborto oscilaría entre 0,8 y 1,6/100.000 nv (2 y 4 muertes por aborto, respectivamente).

¿Cómo es posible lograr en Chile estas bajas cifras de muerte materna por aborto, si en la legislación chilena el aborto es penalizado, independiente de la causa? En general, los países con legislación restrictiva del aborto presentarían altas tasas de aborto ilegal, exponiendo a la población a prácticas abortivas inseguras y de alto riesgo de muerte materna por aborto séptico (16). De acuerdo a las estadísticas analizadas, es poco probable que eso ocurra en Chile. En países donde el aborto es ilegal, se estima que por cada egreso por aborto hay 5 que no se hospitalizan, por lo que en Chile habría anualmente la ya clásica cifra de 160.000 abortos clandestinos, siendo junto a Perú los países que presentarían la incidencia de aborto ilegal más alta en la Región ( 45,4 y $51,8 / 1000$ mujeres, respectivamente) (17). La conclusión de esos análisis es hacer seguro el aborto inseguro, mediante la legalización del aborto, como también mejorar el acceso al aborto seguro de las mujeres con embarazo no deseado, en aquellos países donde el aborto es legal.

Como ya hemos visto, desde la incorporación de los métodos anticonceptivos en el país, la natalidad descendió, con fluctuaciones dependientes de las políticas de salud reproductiva imperantes (18). A partir de 1990, año en que el país alcanzó la natalidad más alta de su historia reproductiva, esta descendió significativamente en $21,2 \%$ en el período 1990-2004, especialmente en el rango de edad materna de 20 a 29 años, registrando además un importante descenso, aunque no significativo, en las adolescentes de 15 a 19 años (19). Esta tendencia es atribuible a la amplia distribución y acceso de las mujeres a los métodos anticonceptivos en el sistema público y privado de salud, como también a la mayor responsabilidad de las mujeres, especialmente de las más jóvenes. Si bien es cierto que el aborto clandestino es una realidad en el país, es injusto pensar que el descenso de la natalidad es producto de ese procedimiento, y no de las políticas actuales 
de salud reproductiva (20). En ningún país el aborto clandestino lleva la natalidad a tasas de fecundidad inferiores a la tasa de recambio poblacional, como sucede en Chile desde el año 2004 (21), ya que eso solo es posible con el progreso social, económico, educacional y sanitario de un país.

Pese a todo lo anterior, la Sociedad Chilena de Obstetricia y Ginecología, propondrá un plan de acción al Ministerio de Salud, dentro de la iniciativa FIGO, que incluye: mayor participación de la Sociedad Chilena de Obstetricia y Ginecología en las políticas públicas de salud reproductiva, estimular el desarrollo de programas de control de la fecundidad en los grupos de riesgos, desarrollar programas de anticoncepción y consejería pos aborto, revisión de las normas técnicas de registro de las muertes maternas, como también el permanente respeto por la vida humana en gestación.

\section{Dr Enrique Donoso S. Editor Jefe. \\ Revista Chilena de Obstetricia y Ginecología.}

\section{BIBLIOGRAFÍA}

1. Faúndes A. La iniciativa FIGO para la prevención del aborto inseguro. Rev Chil Obstet Ginecol 2008;73(4):221-2.

2. Donoso E. Transición demográfica, Chile 1900-2004 (datos no publicados).

3. Viel B, Campos W. Chilean history of infant and maternal mortality, 1940-1985. Perspec Int Planif Fam 1987; (Spec No): 24-8.

4. Donoso E. Mortalidad materna, Chile 2005. Rev Chil Obstet Ginecol 2008;73(3):143-4.

5. Szot J, Moreno C. Mortalidad por aborto en Chile: análisis epidemiológico 1985-2000. Rev Chil Obstet Ginecol 2003;68(4):309-14.

6. Donoso E. Mortalidad materna en Chile: 2000-2004. Rev Chil Obstet Ginecol 2006;71(4):246-51.

7. Donoso E. Plan de Acción Regional para la reducción de la mortalidad materna en las Américas: resultados de Chile. Rev Chil Obstet Ginecol 2003;68(1):13-6.

8. Lewis G, CEMACH. Why mothers die 2000-2002: the confidential enquiries into maternal deaths in the United Kingdom. (Ed: Lewis G). London: RCOG Press;
2004. Hallado en: http://www.cemach.org.uk/Publications/Saving-Mothers-Lives-Report-2000-2002.aspx.

9. Deneux-Tharaux C, Berg C, Bouvier-Colle MH, Gissler M, Harper M, Nannini A, Alexander S, Wildman K, Breart G, Buekens P. Underreporting of pregnancy-related mortality in the United States and Europe. Obstet Gynecol 2005;106(4):684-92.

10. Horon IL. Underreporting of maternal deaths on death certificates and the magnitude of the problem of maternal mortality. Am J Public Health. 2005 Mar;95(3):47882.

11. Szmoisz S, Vuegen SE, Plaza AS, Barracchini R, Checa S, Derlindati A, Espinola DA, Rúgolo EC. Argentina: risk factors and maternal mortality in La Matanza, Province of Buenos Aires, 1990. World Health Stat Q 1995;48(1):4-7.

12. Alves SV. Maternal mortality in Pernambuco,Brazil: what has changed in ten years? Reprod Health Matters 2007;15(30):134-44.

13. McCaw-Binns A, Lindo JL, Lewis-Bell KN, Ashley DE. Maternal mortality surveillance in Jamaica. Int J Gynaecol Obstet. 2008 Jan;100(1):31-6.

14. Mathers CD, Fat DM, Inoue M, Rao C, Lopez AD. Counting the dead and what they died from: an assessment of the global status of cause of death data. Bull World Health Organ 2005;83(3):171-7.

15. WHO. Maternal mortality in 2005: estimated developed by WHO, UNICEF, UNFPA and the World Bank. Hallado en: http://www.who.int/whosis/mme_2005. pdf.

16. The Alan Guttmacher Institute. An overview of clandestine abortion in Latin America. Hallado en: http:// www.guttmacher.org/pubs/ib12.pdf

17. Henshaw SK, Singh S, Haas T. The incidence of abortion worldwide. Int Fam Plann Persp 1999;25(Suppl): S30-8.

18. Aguila A, Muñoz H. Tendencia de la natalidad, mortalidad general, infantil y neonatal en Chile desde el año 1850 a la fecha. Rev Méd Chile 1997;125(10):123645.

19. Donoso E. Descenso de la natalidad en Chile: un problema país. Rev Chil Obstet Ginecol 2007;72(2):73-5.

20. Gobierno de Chile, Ministerio de Salud. Los Objetivos Sanitarios para la década 2000-2010. 1a edición, Octubre 2002. Salud de la Mujer;9-18.

21. Instituto Nacional de Estadísticas. Enfoque Estadístico 2006. Fecundidad en Chile. Hallado en: http://www. ine.cl/canales/sala_prensa/archivo_documentos/enfoques/2006/pdf/fecundidad1.pdf 